\title{
Local Quasitriangular Hopf Algebras
}

\author{
Shouchuan Zhang ${ }^{a, b}$, Mark D. Gould ${ }^{b}$, Yao-Zhong Zhang ${ }^{b}$ \\ a. Department of Mathematics, Hunan University \\ Changsha 410082, P.R. China \\ b. Department of Mathematics, University of Queensland \\ Brisbane 4072, Australia
}

\begin{abstract}
We find a new class of Hopf algebras, local quasitriangular Hopf algebras, which generalize quasitriangular Hopf algebras. Using these Hopf algebras, we obtain solutions of the Yang-Baxter equation in a systematic way. The category of modules with finite cycles over a local quasitriangular Hopf algebra is a braided tensor category.
\end{abstract}

\section{Introduction}

The Yang-Baxter equation first came up in the paper by Yang as factorization condition of the scattering S-matrix in the many-body problem in one dimension and in the work by Baxter on exactly solvable models in statistical mechanics. It has been playing an important role in mathematics and physics ( see [2] , [17] ). Attempts to find solutions of the Yang-Baxter equation in a systematic way have led to the theory of quantum groups and quasitriangular Hopf algebras (see [7]).

Since the category of modules with finite cycles over a local quasitriangular Hopf algebra is a braided tensor category, we may also find solutions of the Yang-Baxter equation in a systematic way.

The main results in this paper are summarized in the following statement.

Theorem 1 (i) Assume that $\left(H,\left\{R_{n}\right\}\right)$ is a local quasitriangular Hopf algebra. Then $\left({ }_{H} \mathcal{M}^{c f}, C^{\left\{R_{n}\right\}}\right),\left({ }_{H} \mathcal{M}^{d c f}, C^{\left\{R_{n}\right\}}\right)$ and $\left({ }_{H} \mathcal{M}^{d f}, C^{\left\{R_{n}\right\}}\right)$ are braided tensor categories. Furthermore, if $\left(M, \alpha^{-}\right)$is an $H$-module with finite cycles and $R_{n+1}=R_{n}+W_{n}$ with $W_{n} \in H_{n+1} \otimes H_{(n+1)}$, then $\left(M, \alpha^{-}, \delta^{-}\right)$is a Yetter-Drinfeld $H$-module. 
(ii) Assume that $B$ is a finite dimensional Hopf algebra and $M$ is a finite dimenaional B-Hopf bimodule. Then $\left(\left(T_{B}(M)\right)^{c o p} \bowtie_{\tau} T_{B^{*}}^{c}\left(M^{*}\right),\left\{R_{n}\right\}\right)$ is a local quasitriangular Hopf algebra. Furthermore, if $\left(k Q^{a}, k Q^{c}\right)$ and $\left(k Q^{s}, k Q^{s c}\right)$ are arrow dual pairings with finite Hopf quiver $Q$, then both $\left(\left(k Q^{a}\right)^{\text {cop }} \bowtie_{\tau} k Q^{c},\left\{R_{n}\right\}\right)$ and $\left(\left(k Q^{s}\right)^{c o p} \bowtie_{\tau} k Q^{s c},\left\{R_{n}\right\}\right)$ are local quasitriangular Hopf algebras.

\section{Preliminaries}

Throughout, we work over a fixed field $k$. All algebras, coalgebras, Hopf algebras, and so on, are defined over $k$. Books $[8,11,16,14]$ provide the necessary background for Hopf algebras and book [1] provides a nice description of the path algebra approach.

Let $V$ and $W$ be two vector spaces. $\sigma_{V}$ denotes the map from $V$ to $V^{* *}$ by defining $<\sigma_{V}(x), f>=<f, x>$ for any $f \in V^{*}, x \in V . C_{V, W}$ denotes the map from $V \otimes W$ to $W \otimes V$ by defining $C_{V, W}(x \otimes y)=y \otimes x$ for any $x \in V, y \in W$. Denote $P$ by $\sum P^{\prime} \otimes P^{\prime \prime}$ for $P \in V \otimes W$. If $V$ is a finite-dimensional vector space over field $k$ with $V^{*}=H_{o m}(V, k)$. Define maps $b_{V}: k \rightarrow V \otimes V^{*}$ and $d_{V}: V^{*} \otimes V \rightarrow k$ by

$$
b_{V}(1)=\sum_{i} v_{i} \otimes v_{i}^{*} \quad \text { and } \quad \sum_{i, j} d_{V}\left(v_{i}^{*} \otimes v_{j}\right)=<v_{i}^{*}, v_{j}>
$$

where $\left\{v_{i} \mid i=1,2, \cdots, n\right\}$ is any basis of $V$ and $\left\{v_{i}^{*} \mid i=1,2, \cdots, n\right\}$ is its dual basis in $V^{*} . d_{V}$ and $b_{V}$ are called evaluation and coevaluation of $V$, respectively. It is clear $\left(d_{V} \otimes i d_{U}\right)\left(i d_{U} \otimes b_{V}\right)=i d_{U}$ and $\left(i d_{V} \otimes d_{V}\right)\left(b_{V} \otimes i d_{V}\right)=i d_{V} . \xi_{V}$ denotes the linear isomorphism from $V$ to $V^{*}$ by sending $v_{i}$ to $v_{i}^{*}$ for $i=1,2, \cdots, n$. Note that we can define evaluation $d_{V}$ when $V$ is infinite.

We will use $\mu$ to denote the multiplication map of an algebra and use $\Delta$ to denote the comultiplication of a coalgebra. For a (left or right) module and a (left or right) comodule, denote by $\alpha^{-}, \alpha^{+}, \delta^{-}$and $\delta^{+}$the left module, right module, left comodule and right comodule structure maps, respectively. The Sweedler's sigma notations for coalgebras and comodules are $\Delta(x)=\sum x_{1} \otimes x_{2}, \delta^{-}(x)=\sum x_{(-1)} \otimes x_{(0)}, \delta^{+}(x)=\sum x_{(0)} \otimes x_{(1)}$. Let $(H, \mu, \eta, \Delta, \epsilon)$ be a bialgebra and let $\Delta^{c o p}:=C_{H, H} \Delta$ and $\mu^{o p}:=\mu C_{H, H}$. We denote $\left(H, \mu, \eta, \Delta^{c o p}, \epsilon\right)$ by $H^{c o p}$ and $\left(H, \mu^{o p}, \eta, \Delta, \epsilon\right)$ by $H^{o p}$. Sometimes, we also denote the unit element of $H$ by $1_{H}$.

Let $A$ and $H$ be two bialgebras with $\emptyset \neq X \subseteq A, \emptyset \neq Y \subseteq H$ and $P \in Y \otimes X, R \in$ $Y \otimes Y$. Assume that $\tau$ is a linear map from $H \otimes A$ to $k$. We give the following notations:

$(Y, R)$ is called almost cocommutative if the following condition satisfied:

$(\mathrm{ACO}): \quad \sum y_{2} R^{\prime} \otimes y_{1} R^{\prime \prime}=\sum R^{\prime} y_{1} \otimes R^{\prime \prime} y_{2}$ for any $y \in Y$.

$\tau$ is called a skew pairing on $H \otimes A$ if for any $x, u \in H, y, z \in A$ the following conditions are satisfied: 
$(\mathrm{SP} 1): \tau(x, y z)=\sum \tau\left(x_{1}, y\right) \tau\left(x_{2}, z\right)$

(SP2): $\tau(x u, z)=\sum \tau\left(x, z_{2}\right) \tau\left(u, z_{1}\right)$;

(SP3): $\tau(x, \eta)=\epsilon_{H}(x) ; \quad(\mathrm{SP} 4): \tau(\eta, y)=\epsilon_{A}(y)$.

$P$ is called a copairing of $Y \otimes X$ if for any $x, u \in H, y, z \in A$ the following conditions are satisfied:

$(\mathrm{CP} 1): \sum P^{\prime} \otimes P_{1}^{\prime \prime} \otimes P_{2}^{\prime \prime}=\sum P^{\prime} Q^{\prime} \otimes Q^{\prime \prime} \otimes P^{\prime \prime}$ with $P=Q$;

(CP1): $\sum P_{1}^{\prime} \otimes P_{2}^{\prime} \otimes P^{\prime \prime}=\sum P^{\prime} \otimes Q^{\prime} \otimes P^{\prime \prime} Q^{\prime \prime}$ with $P=Q$;

(CP3): $\sum P^{\prime} \otimes \epsilon_{A}\left(P^{\prime \prime}\right)=\eta_{H} ; \quad(\mathrm{CP} 4): \sum \epsilon_{H}\left(P^{\prime}\right) \otimes P^{\prime \prime}=\eta_{A}$.

For $R \in H \otimes H$ and two $H$-modules $U$ and $V$, define a linear map $C_{U, V}^{R}$ from $U \otimes V$ to $V \otimes U$ by sending $(x \otimes y)$ to $\sum R^{\prime \prime} y \otimes R^{\prime} x$ for any $x \in U, y \in V$.

If $V=\oplus_{i=0}^{\infty} V_{i}$ is a graded vector space, let $V_{>n}$ and $V_{\leq n}$ denote $\oplus_{i=n+1}^{\infty} V_{i}$ and $\oplus_{i=0}^{n} V_{i}$, respectively. We usually denote $\oplus_{i=0}^{n} V_{i}$ by $V_{(n)}$. If $\operatorname{dim} V_{i}<\infty$ for any natural number $i$, then $V$ is called a local finite graded vector space. We denote by $\iota_{i}$ the natural injection from $V_{i}$ to $V$ and by $\pi_{i}$ the corresponding projection from $V$ to $V_{i}$.

Let $H$ be a bialgebra and a graded coalgebra with an invertible element $R_{n}$ in $H_{(n)} \otimes$ $H_{(n)}$ for any natural $n$. Assume $R_{n+1}=R_{n}+W_{n}$ with $W_{n} \in H_{(n+1)} \otimes H_{n+1}+H_{n+1} \otimes H_{(n+1)}$. ( $\left.H,\left\{R_{n}\right\}\right)$ is called a local quasitriangular bialgebra if $R_{n}$ is a copairing on $H_{(n)} \otimes H_{(n)}$, and $\left(H_{(n)}, R_{n}\right)$ is almost cocommutative for any natural number $n$. In this case, $\left\{R_{n}\right\}$ is called a local quasitriangular structure of $H$. Obviously, if $(H, R)$ is a quasitriangular bialgebra, then $\left(H,\left\{R_{n}\right\}\right)$ is a local quasitriangular bialgebra with $R_{0}=R, R_{i}=0, H_{0}=H, H_{i}=0$ for $i>0$.

The following facts are obvious: $\tau^{-1}=\tau\left(i d_{H} \otimes S\right)\left(\right.$ or $\left.=\tau\left(S^{-1} \otimes i d_{A}\right)\right)$ if $A$ is a Hopf algebra ( or $H$ is a Hopf algebra with invertible antipode ) and $\tau$ is a skew pairing; $P^{-1}=\left(S \otimes i d_{A}\right) P\left(\right.$ or $\left.=\left(i d_{H} \otimes S^{-1}\right) P\right)$ if $H$ is a Hopf algebra ( or $A$ is a Hopf algebra with invertible antipode) and $P$ is a copairing.

Let $A$ be an algebra and $M$ be an $A$-bimodule. Then the tensor algebra $T_{A}(M)$ of $M$ over $A$ is a graded algebra with $T_{A}(M)_{0}=A, T_{A}(M)_{1}=M$ and $T_{A}(M)_{n}=\otimes_{A}^{n} M$ for $n>1$. That is, $T_{A}(M)=A \oplus\left(\bigoplus_{n>0} \otimes_{A}^{n} M\right)$ (see [12]). Let $D$ be another algebra. If $h$ is an algebra map from $A$ to $D$ and $f$ is an $A$-bimodule map from $M$ to $D$, then by the universal property of $T_{A}(M)$ (see [12, Proposition 1.4.1]) there is a unique algebra map $T_{A}(h, f): T_{A}(M) \rightarrow D$ such that $T_{A}(h, f) \iota_{0}=h$ and $T_{A}(h, f) \iota_{1}=f$. One can easily see that $T_{A}(h, f)=h+\sum_{n>0} \mu^{n-1} T_{n}(f)$, where $T_{n}(f)$ is the map from $\otimes_{A}^{n} M$ to $\otimes_{A}^{n} D$ given by $T_{n}(f)\left(x_{1} \otimes x_{2} \otimes \cdots \otimes x_{n}\right)=f\left(x_{1}\right) \otimes f\left(x_{2}\right) \otimes \cdots \otimes f\left(x_{n}\right)$, i.e., $T_{n}(f)=f \otimes_{A} f \otimes_{A} \cdots \otimes_{A} f$. Note that $\mu$ can be viewed as a map from $D \otimes_{A} D$ to $D$. For the details, the reader is directed to [12, Section 1.4].

Dually, let $C$ be a coalgebra and let $M$ be a $C$-bicomodule. Then the cotensor coalgebra $T_{C}^{c}(M)$ of $M$ over $C$ is a graded coalgebra with $T_{C}^{c}(M)_{0}=C, T_{C}^{c}(M)_{1}=M$ 
and $T_{C}^{c}(M)_{n}=\square_{C}^{n} M$ for $n>1$. That is, $T_{C}^{c}(M)=C \oplus\left(\bigoplus_{n>0} \square_{C}^{n} M\right)$ (see [12]). Let $D$ be another coalgebra. If $h$ is a coalgebra map from $D$ to $C$ and $f$ is a $C$ bicomodule map from $D$ to $M$ such that $f(\operatorname{corad}(D))=0$, then by the universal property of $T_{C}^{c}(M)$ (see [12, Proposition 1.4.2]) there is a unique coalgebra map $T_{C}^{c}(h, f)$ from $D$ to $T_{C}^{c}(M)$ such that $\pi_{0} T_{C}^{c}(h, f)=h$ and $\pi_{1} T_{C}^{c}(h, f)=f$. It is not difficult to see that $T_{C}^{c}(h, f)=h+\sum_{n>0} T_{n}^{c}(f) \Delta_{n-1}$, where $T_{n}^{c}(f)$ is the map from $\square_{C}^{n} D$ to $\square_{C}^{n} M$ induced by $T_{n}(f)\left(x_{1} \otimes x_{2} \otimes \cdots \otimes x_{n}\right)=f\left(x_{1}\right) \otimes f\left(x_{2}\right) \otimes \cdots \otimes f\left(x_{n}\right)$, i.e., $T_{n}^{c}(f)=f \otimes f \otimes \cdots \otimes f$.

Furthermore, if $B$ is a Hopf algebra and $M$ is a $B$-Hopf bimodule, then $T_{B}(M)$ and $T_{B}^{c}(M)$ are two graded Hopf algebra. Indeed, by [12, Section 1.4] and [12, Proposition 1.5.1], $T_{B}(M)$ is a graded Hopf algebra with the counit $\varepsilon=\varepsilon_{B} \pi_{0}$ and the comultiplication $\Delta=\left(\iota_{0} \otimes \iota_{0}\right) \Delta_{B}+\sum_{n>0} \mu^{n-1} T_{n}\left(\Delta_{M}\right)$, where $\Delta_{M}=\left(\iota_{0} \otimes \iota_{1}\right) \delta_{M}^{-}+\left(\iota_{1} \otimes \iota_{0}\right) \delta_{M}^{+}$. Dually, $T_{B}^{c}(M)$ is a graded Hopf algebra with multiplication $\mu=\mu_{B}\left(\pi_{0} \otimes \pi_{0}\right)+\sum_{n>0} T_{n}^{c}\left(\mu_{M}\right) \Delta_{n-1}$, where $\mu_{M}=\alpha_{M}^{-}\left(\pi_{0} \otimes \pi_{1}\right)+\alpha_{M}^{+}\left(\pi_{1} \otimes \pi_{0}\right)$.

\section{Yang-Baxter Equations}

Assume that $H$ is a bialgebra and a graded coalgebra with an invertible element $R_{n}$ in $H_{(n)} \otimes H_{(n)}$ for any natural $n$. For convenience, let (LQT1), (LQT2) and (LQT3) denote $(\mathrm{CP} 1),(\mathrm{CP} 2)$ and $(\mathrm{ACO})$, respectively;

(LQT4): $R_{n+1}=R_{n}+W_{n}$ with $W_{n} \in H_{(n+1)} \otimes H_{n+1}+H_{n+1} \otimes H_{(n+1)}$;

(LQT4'): $R_{n+1}=R_{n}+W_{n}$ with $W_{n} \in H_{n+1} \otimes H_{n+1}$.

Then $\left(H,\left\{R_{n}\right\}\right)$ is a local quasitriangular bialgebra iff (LQT1), (LQT2), (LQT3) and (LQT4) hold for any natural number $n$.

Let $H$ be a graded coalgebra and a bialgebra. A left $H$-module $M$ is called an $H$ module with finite cycles if, for any $x \in M$, there exists a natural number $n_{x}$ such that $H_{i} x=0$ when $i>n_{x}$. Let ${ }_{H} \mathcal{M}^{c f}$ denote the category of all left $H$-modules with finite cycles.

Lemma 1.1 Let $H$ be a graded coalgebra and a bialgebra. If $U$ and $V$ are left $H$ modules with finite cycles, so is $U \otimes V$.

Proof. For any $x \in U, y \in V$, there exist two natural numbers $n_{x}$ and $n_{y}$, such that $H_{>n_{x}} x=0$ and $H_{>n_{y}} y=0$. Set $n_{x \otimes y}=2 n_{x}+2 n_{y}$. It is clear that $H_{>n_{x \otimes y}}(x \otimes y)=0$. Indeed, for any $h \in H_{i}$ with $i>n_{x \otimes y}$, we see

$$
\begin{aligned}
h(x \otimes y) & =\sum h_{1} x \otimes h_{2} y \\
& =0 \quad(\text { since } H \text { is graded coalgebra }) .
\end{aligned}
$$


Lemma 1.2 Assume that $\left(H,\left\{R_{n}\right\}\right)$ is a local quasitriangular Hopf algebra. Then for any left $H$-modules $U$ and $V$ with finite cycles, there exists an invertible linear map $C_{U, V}^{\left\{R_{n}\right\}}: U \otimes V \rightarrow V \otimes U$ such that

$$
C_{U, V}^{\left\{R_{n}\right\}}(x \otimes y):=C^{R_{n}}(x \otimes y)=\sum R_{n}^{\prime \prime} y \otimes R_{n}^{\prime} x
$$

with $n>2 n_{x}+2 n_{y}$, for $x \in U, y \in V$.

Proof. We first define a map $f$ from $U \times V$ to $V \otimes U$ by sending $(x, y)$ to $\sum R_{n}^{\prime \prime} y \otimes R_{n}^{\prime} x$ with $n>2 n_{x}+2 n_{y}$ for any $x \in U, y \in V$. It is clear that $f$ is well defined. Indeed, if $n>2 n_{x}+2 n_{y}$, then $C^{R_{n+1}}(x \otimes y)=C^{R_{n}}(x \otimes y)$ since $R_{n+1}=R_{n}+W_{n}$ with $W_{n} \in H_{(n+1)} \otimes$ $H_{n+1}+H_{n+1} \otimes H_{(n+1)} \cdot f$ is a $k$-balanced function. Indeed, for $x, y \in U, z, w \in V, \alpha \in k$, let $n>2 n_{x}+2 n_{y}+2 n_{z}$. See

$$
\begin{aligned}
f(x+y, z) & =\sum R_{n}^{\prime \prime} z \otimes R_{n}^{\prime}(x+y) \\
& =\sum R_{n}^{\prime \prime} z \otimes R_{n}^{\prime} x+\sum R_{n}^{\prime \prime} z \otimes R_{n}^{\prime} y \\
& =f(x, z)+f(y, z) .
\end{aligned}
$$

Similarly, we can show that $f(x, z+w)=f(x, z)+f(x, w), f(x \alpha, z)=f(x, \alpha z)$. Consequently, there exists a linear map $C_{U, V}^{\left\{R_{n}\right\}}: U \otimes V \rightarrow V \otimes U$ such that

$$
C_{U, V}^{\left\{R_{n}\right\}}(x \otimes y)=C^{R_{n}}(x \otimes y)
$$

with $n>2 n_{x}+2 n_{y}$, for $x \in U, y \in V$.

The inverse $\left(C_{U, V}^{\left\{R_{n}\right\}}\right)^{-1}$ of $C_{U, V}^{\left\{R_{n}\right\}}$ is defined by sending $(y \otimes x)$ to $\sum\left(R_{n}^{-1}\right)^{\prime} x \otimes\left(R_{n}^{-1}\right)^{\prime \prime} y$ with $n>2 n_{x}+2 n_{y}$ for any $x \in U, y \in V$.

Theorem 2 Assume that $\left(H,\left\{R_{n}\right\}\right)$ is a local quasitriangular Hopf algebra. Then $\left({ }_{H} \mathcal{M}^{c f}, C^{\left\{R_{n}\right\}}\right)$ is a braided tensor category.

Proof. Since $H$ is a bialgebra, we have that $\left({ }_{H} \mathcal{M}, \otimes, I, a, r, l\right)$ is a tensor category by [15, Proposition XI. 3.1]. It follows from Lemma 1.1 that $\left({ }_{H} \mathcal{M}^{c f}, \otimes, I, a, r, l\right)$ is a tensor subcategory of $\left({ }_{H} \mathcal{M}, \otimes, I, a, r, l\right) . C^{\left\{R_{n}\right\}}$ is a braiding of ${ }_{H} \mathcal{M}^{c f}$, which can be shown by the way similar to proof of [15, Proposition VIII. 3.1, Proposition XIII. 1.4].

An $H$-module $M$ is called a graded $H$-module if $M=\oplus_{i=0}^{\infty} M_{i}$ is a graded vector space and $H_{i} M_{j} \subseteq M_{i+j}$ for any natural number $i$ and $j$.

Lemma 1.3 Assume that $H$ is a local finite graded coalgebra and bialgebra. If $M=$ $\oplus_{i=0}^{\infty} M_{i}$ is a graded $H$-module, then the following conditions are equivalent:

(i) $M$ is an $H$-module with finite cycles.

(ii) $H x$ is finite dimensional for any $x \in M$

(iii) $H x$ is finite dimensional for any homogeneous element $x$ in $M$. 
Proof. (i) $\Rightarrow$ (ii). For any $x \in M$, there exists a natural number $n_{x}$ such that $H_{i} x=$ with $i>n_{x}$. Since $H /(0: x)_{H} \cong H x$, where $(0: x)_{H}:=\{h \in H \mid h \cdot x=0\}$, we have that $H x$ is finite dimensional.

(ii) $\Rightarrow$ (iii). It is clear.

(iii) $\Rightarrow$ (i). We first show that, for any homogeneous element $x \in M_{i}$, there exists a natural number $n_{x}$ such that $H_{j} x=0$ with $j>n_{x}$. In fact, if the above does not hold, then there exists $h_{j} \in H_{n_{j}}$ such that $h_{j} x \neq 0$ with $n_{1}<n_{2}<\cdots$. Considering $h_{j} x \in M_{n_{j}+i}$ we have that $\left\{h_{j} x \mid j=1,2, \cdots\right\}$ is linear independent in $H x$, which contradicts to that $H x$ is finite dimensional.

For any $x \in M$, then $x=\sum_{i=1}^{l} x_{i}$ and $x_{i}$ is a homogeneous element for $i=1,2, \cdots, l$. There exists a natural number $n_{x_{i}}$ such that $H_{j} x_{i}=0$ with $j>n_{x_{i}}$. Set $n_{x}=\sum_{s=1}^{l} n_{x_{s}}$. Then $H_{j} x=0$ with $j>n_{x}$. Consequently, $M$ is an $H$-module with finite cycles.

Note that if $M=\oplus_{i=0}^{\infty} M_{i}$ is a graded $H$-module, then both (ii) $\Rightarrow$ (iii) and (iii) $\Rightarrow$ (i) hold in Lemma 1.3.

Let ${ }_{H} \mathcal{M}^{g f}$ and ${ }_{H} \mathcal{M}^{g c f}$ denote the category of all finite dimensional graded left $H$ modules and the category of all graded left $H$-modules with finite cycles. Obviously, they are two tensor subcategories of ${ }_{H} \mathcal{M}^{c f}$. Therefore we have

Theorem 3 Assume that $\left(H,\left\{R_{n}\right\}\right)$ is a local quasitriangular Hopf algebra. Then $\left({ }_{H} \mathcal{M}^{g f}, C^{\left\{R_{n}\right\}}\right)$ and $\left({ }_{H} \mathcal{M}^{g c f}, C^{\left\{R_{n}\right\}}\right)$ are two braided tensor categories.

Therefore, if $M$ is a finite dimensional graded $H$ - module (or $H$-module with finit cycles) over local quasitriangular Hopf algebra $\left(H,\left\{R_{n}\right\}\right)$, then $C_{M, M}^{\left\{R_{n}\right\}}$ is a solution of Yang-Baxter equation on $M$.

It is easy to prove the following.

Theorem 4 Assume that $\left(H,\left\{R_{n}\right\}\right)$ is a local quasitriangular Hopf algebra and $R_{n+1}=R_{n}+W_{n}$ with $W_{n} \in H_{n+1} \otimes H_{(n+1)}$. If $\left(M, \alpha^{-}\right)$is an H-modules with finite cycles then $\left(M, \alpha^{-}, \delta^{-}\right)$is a Yetter-Drinfeld $H$-module, where $\delta^{-}(x)=\sum R_{n}^{\prime \prime} \otimes R_{n}^{\prime} x$ for any $x \in M$ and $n \geq n_{x}$.

\section{The Relation between Tensor Algebras and Co- tensor Coalgebras}

Lemma 2.1 (See [3, 8]) Let $A, B$ and $C$ be finite dimensional coalgebras, $\left(M, \delta_{M}^{-}, \delta_{M}^{+}\right)$ and $\left(N, \delta_{N}^{-}, \delta_{N}^{+}\right)$be respectively a finite dimensional $A$-B-bicomodule and a finite dimensional B-C-bicomodule. Then

(i) $\left(M^{*}, \delta_{M}^{-*}, \delta_{M}^{+*}\right)$ is a finite dimensional $A^{*}-B^{*}$-bimodule. 
(ii) $\left(M \square_{B} N, \delta_{M \square_{B} N}^{-}, \delta_{M \square_{B} N}^{+}\right)$is an A-C-bicomodule with structure maps $\delta_{M \square_{B} N}^{-}=$ $\delta_{M}^{-} \otimes i d_{N}$ and $\delta_{M \square_{B} N}^{+}=i d_{M} \otimes \delta_{N}^{+}$.

(iii) $M^{*} \otimes_{B^{*}} N^{*} \cong\left(M \square_{B} N\right)^{*}$ (as $A^{*}$-C $C^{*}$-bimodules).

Proof. (i) is obvious.

(ii) For any $m \in M, n \in N$ satisfying $m \otimes n \in M \square_{B} N$, we have that $\left(\delta_{M \square N}^{-}\right)(m \otimes n)=$ $\left(\delta_{M}^{-} \otimes i d_{N}\right)(m \otimes n)=\sum_{i=1}^{l} u_{i} \otimes v_{i} \otimes n$, where $\delta_{M}^{-}(m)=\sum_{i=1}^{l} u_{i} \otimes v_{i}$, and $u_{1}, u_{2}, \cdots, u_{l}$ are linearly independent. We now show that for $i=1,2, \cdots, l$ we have $v_{i} \otimes n \in M \square_{B} N$. Consider

$$
\begin{aligned}
& \left(i d \otimes \delta_{M}^{+} \otimes i d\right)\left(\delta_{M}^{-} \otimes i d\right)(m \otimes n) \\
= & \left(\delta_{M}^{-} \otimes i d \otimes i d\right)\left(\delta_{M}^{+} \otimes i d\right)(m \otimes n) \quad(\text { since } M \text { is an } A-B \text { - bicomodule. }) \\
= & \left(\delta_{M}^{-} \otimes i d \otimes i d\right)\left(i d \otimes \delta_{N}^{-}\right)(m \otimes n) \quad\left(\text { since } m \otimes n \in M \square_{B} N\right) .
\end{aligned}
$$

Therefore,

$$
\sum_{i=1}^{l} \sum_{\left(v_{i}\right)} u_{i} \otimes\left(v_{i}\right)_{(0)} \otimes\left(v_{i}\right)_{(1)} \otimes n=\sum_{i=1}^{l} \sum_{(n)} u_{i} \otimes v_{i} \otimes n_{(-1)} \otimes n_{(0)} .
$$

Since $u_{1}, u_{2}, \cdots, u_{l}$ are linearly independent, we have that $v_{i} \otimes n \in M \square_{B} N$, which shows that $\delta_{M \square N}^{-}$is a map from $M \square_{B} N$ to $A \otimes M \square_{B} N$ (indeed, the fact is given in [8, Page 86$87])$. In this way, we can finish the proof easily.

(iii) It has been known that $\rho\left(f^{*} \otimes g^{*}\right)(m \times n)=f^{*}(m) g^{*}(n)$ gives a $k$-linear isomorphism from $M^{*} \otimes N^{*}$ to $(M \otimes N)^{*}$. We will regard $\left(M^{*} \otimes N^{*}\right)$ and $(M \otimes N)^{*}$ as the same vector space. For any $f^{*} \in M^{*}, g^{*} \in N^{*}, b^{*} \in B^{*}, m \otimes n \in M \square_{B} N$, we consider

$$
\begin{aligned}
\rho\left(f^{*} b^{*} \otimes g^{*}\right)(m \otimes n) & =\left(f^{*} b^{*}\right)(m) g^{*}(n) \\
& =f^{*}\left(b^{*} \cdot m\right) g^{*}(n) \\
& =f^{*}(m)\left(b^{*} g^{*}\right)(n) \quad\left(\text { since } m \otimes n \in M \square_{B} N\right) \\
& =\left(f^{*} \otimes b^{*} g^{*}\right)(m \otimes n) .
\end{aligned}
$$

Thus $\rho$ can be lifted to a $k$-linear morphism $\bar{\rho}$ from $M^{*} \otimes_{B^{*}} N^{*}$ to $\left(M \square_{B} N\right)^{*}$ by sending $f^{*} \otimes_{B^{*}} g^{*}$ to $f^{*} \otimes_{k} g^{*}$ for any $f^{*} \in M^{*}, g^{*} \in N^{*}$. We now show that $\bar{\rho}$ is bijective.

Let $M \otimes N=W \oplus M \square_{B} N$ as vector spaces. We have that

$$
(M \otimes N)^{*} / W^{*} \cong\left(M \square_{B} N\right)^{*} \quad \text { (as vector spaces) }
$$

Set $I=<\left\{f^{*} b^{*} \otimes g^{*}-f^{*} \otimes b^{*} g^{*} \mid f^{*} \in M^{*}, g^{*} \in N^{*}, b^{*} \in B^{*}\right\}>$, i.e. $I$ is a subspace spanned by $\left\{f^{*} b^{*} \otimes g^{*}-f^{*} \otimes b^{*} g^{*} \mid f^{*}, M^{*}, g^{*} \in N^{*}, b^{*} \in B^{*}\right\}$ in $M^{*} \otimes N^{*}$. We now show that $I=W^{*}$. For any $f^{*} \in M^{*}, g^{*} \in N^{*}, b^{*} \in B^{*}, m \otimes n \in M \square_{B} N$, by (1), it is 
obtained that $f^{*} b^{*} \otimes g^{*}-f^{*} \otimes b^{*} g^{*} \in W^{*}$. Thus $I \subseteq W^{*}$. Conversely, for any $u \in W^{*}$ and $u \in(M \otimes N)^{*}$, then $u\left(M \square_{B} N\right)=0$. Let $\zeta=\delta_{M}^{+} \otimes i d_{N}-i d_{M} \otimes \delta_{N}^{-}$. Since ker $\zeta \subseteq k e r u$, there exists $\bar{u}: \operatorname{Im}(\zeta) \rightarrow k$ such that $\bar{u} \zeta=u$, i.e. $\zeta^{*}(\bar{u})=u$. Clearly, there exist $m_{i}^{*} \in M^{*}, b_{i}^{*} \in B^{*}, n_{i}^{*} \in N^{*}$ such that $\bar{u}=\sum m_{i}^{*} \otimes b_{i}^{*} \otimes n_{i}^{*}$. Therefore,

$$
\begin{aligned}
u & =\sum_{\zeta^{*}}\left(m_{i}^{*} \otimes b_{i}^{*} \otimes n_{i}^{*}\right) \\
& =\sum_{i}\left(m_{i}^{*} b_{i}^{*} \otimes n_{i}^{*}-m_{i}^{*} \otimes b_{i}^{*} n_{i}^{*}\right),
\end{aligned}
$$

which shows that $u \in I$. So $I=W^{*}$.

We now show that $\bar{\rho}$ is surjective. Since for any $v \in\left(M \square_{B} N\right)^{*}$ there exist $m_{i}^{*} \in$ $M^{*}, n_{i}^{*} \in N^{*}$ such that $v=\sum m_{i}^{*} \otimes n_{i}^{*}$, we have $v=\bar{\rho} \sum\left(m_{i}^{*} \otimes_{B^{*}} n_{i}^{*}\right)$, which shows that $\bar{\rho}$ is surjective. So $\bar{\rho}$ is bijective.

Finally, we can check directly that $\bar{\rho}$ is an $A^{*}-C^{*}$-bimodule map.

Lemma 2.2 (See $[3,8]$ ) Let $A, B$ and $C$ be finite dimensional algebras, $\left(M, \alpha_{M}^{-}, \alpha_{M}^{+}\right)$ and $\left(N, \alpha_{N}^{-}, \alpha_{N}^{+}\right)$be respectively a finite dimensional $A$-B-bimodule and a finite dimensional B-C-bimodule. Then

(i) $\left(M^{*}, \alpha_{M}^{-}, \alpha_{M}^{+*}\right)$ is a finite dimensional $A^{*}-B^{*}$-bicomodule.

(ii) $\left(M \otimes_{B} N, \alpha_{M \otimes_{B} N}^{-}, \alpha_{M \otimes_{B} N}^{+}\right)$is an A-C-bimodule with structure maps $\alpha_{M \otimes_{B} N}^{-}=$ $\alpha_{M}^{-} \otimes i d_{N}$ and $\alpha_{M \otimes_{B} N}^{+}=i d_{M} \otimes \alpha_{N}^{+}$.

(iii) $M^{*} \square_{B^{*}} N^{*} \cong\left(M \otimes_{B} N\right)^{*} \quad$ (as $A^{*}-C^{*}$-bicomodules).

Proof.(i) and (ii) are easy.

(iii) Consider

$$
\begin{aligned}
M^{*} \square_{B^{*}} N^{*} & \cong\left(M^{*} \square_{B^{*}} N^{*}\right)^{* *} \\
& \cong\left(M \otimes_{B} N\right)^{*} \quad(\text { by Lemma } 2.1) .
\end{aligned}
$$

Of course, we can also prove it in the dual way of the proof of Lemma2.1, by sending $f \otimes_{k} g$ to $f \otimes_{B^{*}} g$ for any $f \in M^{*}, g \in N^{*}$ with $f \otimes g \in M^{*} \square_{B^{*}} N^{*}$.

Theorem 5 Let $A$ be a finite dimensional algebra and $M$ a finite dimensional $A$ - bimodule. Then $T_{A}(M)$ is isomorphic to subalgebra $\sum_{n=0}^{\infty}\left(\square_{A^{*}}^{n} M^{*}\right)^{*}$ of $\left(T_{A^{*}}^{c}\left(M^{*}\right)\right)^{0}$. Furthermore, this isomorphism is $\sigma_{T_{A}(M)}$ and $\sigma_{T_{A}(M)}=\sigma_{A}+\sum_{n>0} \mu^{n-1} T_{n}\left(\sigma_{M}\right)$ with $\mu^{n-1} T_{n}\left(\sigma_{M}\right)=$ $\sigma_{\otimes_{A}^{n} M}$

Proof. We view $\oplus_{n=0}^{\infty}\left(\square_{A^{*}}^{n} M^{*}\right)^{*}$ as inner direct sum of vector spaces. It is clear that $\sigma_{A}$ is algebra homomorphism from $A$ to $A^{* *} \subseteq\left(T_{A^{*}}^{c}\left(M^{*}\right)\right)^{*}$ and $\sigma_{M}$ is a $A$-bimodule homomorphism from $M$ to $M^{* *} \subseteq\left(T_{A^{*}}^{c}\left(M^{*}\right)\right)^{*}$. Thus it follows from [12, Proposition 
1.4.1] that $\phi=\sigma_{A}+\sum_{n>0} \mu^{n-1} T_{n}\left(\sigma_{M}\right)$ is an algebra homomorphism from $T_{A}(M)$ to $\left(T_{A^{*}}^{c}\left(M^{*}\right)\right)^{*}$.

It follows from Lemma 2.1 (iii) that $\mu^{n-1} T_{n}\left(\sigma_{M}\right)=\sigma_{\otimes_{A}^{n} M}$. Indeed, we use induction on $n>0$. Obviously, the conclusion holds when $n=1$. Let $n>1, N=\otimes_{A}^{n-1} M$, $L=\left(\square_{A^{*}}^{n-1} M^{*}\right)^{*}$ and $\zeta=\mu^{n-2} T_{n-1}\left(\sigma_{M}\right)$. Obviously, $\mu^{n-1} T_{n}\left(\sigma_{M}\right)=\mu\left(\zeta \otimes \sigma_{M}\right)$. By inductive assumption, $\zeta=\sigma_{N}$ is an $A$-bimodule isomorphism from $N$ to $L$. See

$$
\begin{aligned}
\otimes_{A}^{n} M=N \otimes_{A} M & \stackrel{\nu_{1}}{\cong} L \otimes_{A} M^{* *} \text { ( by inductive assumption ) } \\
& \stackrel{\nu_{2}}{\cong}\left(\square_{A^{*}}^{n-1} M^{*}\right)^{*} \otimes_{A^{* *}} M^{* *} \\
& \stackrel{\nu_{3}}{\cong}\left(\left(\square_{A^{*}}^{n-1} M^{*}\right) \square_{A^{*}} M^{*}\right)^{*} \quad(\text { by Lemma } 2.1 \text { (iii) }) \\
& =\left(\square_{A^{*}}^{n} M^{*}\right)^{*},
\end{aligned}
$$

where $\nu_{1}=\sigma_{N} \otimes_{A} \sigma_{M}, \nu_{2}\left(f^{* *} \otimes_{A} g^{* *}\right)=f^{* *} \otimes_{A^{* *}} g^{* *}$ and $\nu_{3}\left(f^{* *} \otimes_{A^{* *}} g^{* *}\right)=f^{* *} \otimes_{k} g^{* *}$ for any $f^{* *} \in\left(\square_{A^{*}}^{n-1} M^{*}\right)^{*}, g^{* *} \in M^{* *}$. Now we have to show $\nu_{3} \nu_{2} \nu_{1}=\sigma_{\otimes_{A}^{n} M}=\mu^{n-1} T_{n}\left(\sigma_{M}\right)$. For any $f^{*} \in \square_{A^{*}}^{n-1} M^{*}, g^{*} \in M^{*}, x \in \otimes_{A}^{n-1} M, y \in M$, on the one hand

$$
<\sigma_{\otimes_{A}^{n} M}\left(x \otimes_{A} y\right), f^{*} \otimes_{k} g^{*}>=<f^{*}, x><g^{*}, y>.
$$

On the other hand,

$$
\begin{aligned}
<\nu_{3} \nu_{2} \nu_{1}\left(x \otimes_{A} y\right), f^{*} \otimes_{k} g^{*}>= & <\nu_{3} \nu_{2}\left(\sigma_{N}(x) \otimes_{A} \sigma_{M}(y)\right), f^{*} \otimes_{k} g^{*}> \\
= & <\sigma_{M}(x) \otimes_{k} \sigma_{N}(y), f^{*} \otimes_{k} g^{*}> \\
= & <f^{*}, x><g^{*}, y>.
\end{aligned}
$$

Thus $\nu_{3} \nu_{2} \nu_{1}=\sigma_{\otimes_{A}^{n} M}$. See

$$
\begin{aligned}
<\mu\left(\zeta \otimes_{A} \sigma_{M}\right)\left(x \otimes_{A} y\right), f^{*} \otimes_{k} g^{*}> & =<\zeta(x) \otimes_{A} \sigma_{M}(y), \Delta\left(f^{*} \otimes_{k} g^{*}\right)> \\
= & <\zeta(x), f^{*}><\sigma_{M}(y), g^{*}> \\
= & <f^{*}, x><g^{*}, y>.
\end{aligned}
$$

Thus $\sigma_{\otimes_{A}^{n} M}=\mu^{n-1} T_{n}\left(\sigma_{M}\right)$.

Finally, for any $x \in T_{A}(M)$ with $x=x^{(1)}+x^{(2)}+\cdots+x^{(n)}$ and $x^{(i)} \in \otimes_{A}^{i} M$,

$$
\begin{aligned}
\phi(x) & =\sum_{i=1}^{n} \phi\left(x^{(i)}\right) \\
& =\sum_{i=1}^{n} \sigma_{\otimes_{A}^{i} M}\left(x^{(i)}\right) \\
& =\sigma_{T_{A}(M)}(x) .
\end{aligned}
$$




\section{3 (Co-)Tensor Hopf Algebras}

Lemma 3.1 Assume that $B$ is a finite dimensional Hopf algebra and $M$ is a finite dimenaional $B$-Hopf bimodule. Let $A:=T_{B}(M)^{c o p}, H:=T_{B^{*}}^{c}\left(M^{*}\right)$. Then

(i) $\phi:=\sigma_{B}+\sum_{i>0} \mu^{i-1} T_{i}\left(\sigma_{M}\right)$ is a Hopf algebra isomorphism from $T_{B}(M)$ to the Hopf subalgebra $\sum_{i=0}^{\infty}\left(\square_{B^{*}}^{i} M^{*}\right)^{*}$ of $\left(T_{B^{*}}^{c}\left(M^{*}\right)\right)^{0}$;

(ii) Let $\phi_{n}:=\left.\phi\right|_{A_{(n)}}$ for any natural number $n \geq 0$. Then there exists $\psi_{n}:\left(H_{(n)}\right)^{*} \rightarrow$ $A_{(n)}$ such that $\phi_{n} \psi_{n}=i d_{\left(H_{(n)}\right)}$ and $\psi_{n} \phi_{n}=i d_{A_{(n)}}$, and $\psi_{n+1}(x)=\psi_{n}(x)$ for any $x \in$ $\left(H_{(n)}\right)^{*}$. Furthermre, $\phi_{n}$ and $\psi_{n}$ preserve the (co)multiplication operations of $T_{B}(M)$ and $\left(T_{B^{*}}^{c}\left(M^{*}\right)\right)^{0}$, respectively.

Proof. (i) We first show that $\left(\square_{B^{*}}^{n} M^{*}\right)^{*} \subseteq\left(T_{B^{*}}^{c}\left(M^{*}\right)\right)^{0}$. For any $f \in\left(\square_{B^{*}}^{n} M^{*}\right)^{*}$, $\sum_{i=n+1}^{\infty} \square_{B^{*}}^{i} M^{*} \subseteq \operatorname{kerf}$ and $\sum_{i=n+1}^{\infty} \square_{B^{*}}^{i} M^{*}$ is a finite codimensional ideal of $T_{B^{*}}^{c}\left(M^{*}\right)$. Consequently, $f \in\left(T_{B^{*}}^{c}\left(M^{*}\right)\right)^{0}$.

Next we show that $\phi:=\sigma_{B}+\sum_{n>0} \mu^{n-1} T_{n}\left(\sigma_{M}\right)$ (see the proof of Theorem 5) is a coalgebra homomorphism from $T_{B}(M)$ to $\sum_{i=0}^{\infty}\left(\square_{B^{*}}^{c} M^{*}\right)^{*}$. For any $x \in \otimes_{B}^{n} M, f, g \in$ $T_{B^{*}}^{c}\left(M^{*}\right)$, on the one hand

$$
\begin{aligned}
<\phi(x), f * g> & =<f * g, x>\quad(\text { by Theorem 5) } \\
& =\sum_{x}<f, x_{1}><g, x_{2}> \\
& =\sum_{x}<\phi\left(x_{1}\right), f><\phi\left(x_{2}\right), g>.
\end{aligned}
$$

On the other hand

$$
<\phi(x), f * g>=\sum<(\phi(x))_{1}, f><(\phi(x))_{2}, g>
$$

since $\phi(x) \in\left(T_{B^{*}}^{c}\left(M^{*}\right)\right)^{0}$. Considering $T_{B^{*}}^{c}\left(M^{*}\right)=\oplus_{n \geq 0} \square_{B^{*}}^{n} M^{*} \cong \oplus_{n \geq 0}\left(\otimes_{B}^{n} M\right)^{*}$ as vector spaces, we have that $T_{B^{*}}\left(M^{*}\right)$ is dense in $\left(T_{B}(M)\right)^{*}$. Consequently, $\sum \phi\left(x_{1}\right) \otimes \phi\left(x_{2}\right)=$ $\sum(\phi(x))_{1} \otimes(\phi(x))_{2}$, i.e. $\phi$ is a coalgebra homomorphism.

(ii) It follows from Theorem 5 .

Recall the double cross product $A_{\alpha} \bowtie_{\beta} H$, defined in [18, P36]) and [15, Definition IX.2.2]. Assume that $H$ and $A$ are two bialgebras; $(A, \alpha)$ is a left $H$-module coalgebra and $(H, \beta)$ is a right $A$-module coalgebra. We define the multiplication $m_{D}$, unit $\eta_{D}$, comultiplication $\Delta_{D}$ and counit $\epsilon_{D}$ in $A \otimes H$ as follows:

$\mu_{D}((a \otimes h) \otimes(b \otimes g))=\sum a \alpha\left(h_{1}, b_{1}\right) \otimes \beta\left(h_{2}, b_{2}\right) g, \quad \Delta_{D}(a \otimes h)=\sum\left(a_{1} \otimes h_{1} \otimes a_{2} \otimes h_{2}\right)$, 
$\epsilon_{D}=\epsilon_{A} \otimes \epsilon_{H}, \eta_{D}=\eta_{A} \otimes \eta_{H}$. for any $a, b \in A, h, g \in H$. We denote $\left(A \otimes H, \mu_{D}, \eta_{D}, \Delta_{D}, \epsilon_{D}\right)$ by

$$
A_{\alpha} \bowtie_{\beta} H,
$$

which is called the double cross product of $A$ and $H$.

Lemma 3.2 (See [9]) Let $H$ and $A$ be two bialgebras. Assume that $\tau$ is an invertible skew pairing on $H \otimes A$. If we define $\alpha(h, a)=\sum \tau\left(h_{1}, a_{1}\right) a_{2} \tau^{-1}\left(h_{2}, a_{3}\right)$ and $\beta(h, a)=$ $\sum \tau\left(h_{1}, a_{1}\right) h_{2} \tau^{-1}\left(h_{3}, a_{2}\right)$ then the double cross product $A_{\alpha} \bowtie_{\beta} H$ of $A$ and $H$ is a bialgebra. Furthermore, if $A$ and $H$ are two Hopf algebras, then so is $A_{\alpha} \bowtie_{\beta} H$.

Proof. We can check that $(A, \alpha)$ is an $H$-module coalgebra and $(H, \beta)$ is an $A$-module coalgebra step by step. We can also check that (M1)-(M4) in [18, P36-37] hold step by step. Consequently, it follows from [18, Corollary 1.8, Theorem 1.5] or [15, Theorem IX.2.3] that $A_{\alpha} \bowtie_{\beta} H$ is a Hopf algebra.

In this case, $A_{\alpha} \bowtie_{\beta} H$ can be written as $A \bowtie_{\tau} H$.

Lemma 3.3 Let $H$ and $A$ be two Hopf algebra. Assume that there exists a Hopf algebra monomorphism $\phi: A^{\text {cop }} \rightarrow H^{0}$. Set $\tau=d_{H}\left(\phi \otimes i d_{H}\right) C_{H, A}$. Then $A \bowtie_{\tau} H$ is Hopf algebra.

Proof. Using [10, Proposition 2.4] or the definition of the evaluation and coevaluation on tensor product, we can obtain that $\tau$ is a skew pairing on $H \otimes A$. Considering Lemma 3.2 , we complete the proof.

Lemma 3.4 (i) If $H=\oplus_{n=0}^{\infty} H_{n}$ is a graded bialgebra and $H_{0}$ has an invertible antipode, then $H$ has an invertible antipode.

(ii) Assume that $B$ is a finite dimensional Hopf algebra and $M$ is a B-Hopf bimodule. Then both $T_{B}(M)$ and $T_{B}^{c}(M)$ have invertible antipodes.

Proof. (i) It is clear that $H^{o p}$ is a graded bialgebra with $\left(H^{o p}\right)_{0}=\left(H_{0}\right)^{(o p)}$. Thus $H^{o p}$ has an antipode by [12, Proposition 1.5.1]. However, the antipode of $H^{o p}$ is the inverse of antipode of $H$.

(ii) It follows from (i).

Lemma 3.5 Let $A=\oplus_{n=0}^{\infty} A_{n}$ and $H=\oplus_{n=0}^{\infty} H_{n}$ be two graded Hopf algebras with invertible antipodes. Let $\tau$ be a skew pairing on $(H \otimes A)$ and $P_{n}$ be a copairing of $H_{(n)} \otimes A_{(n)}$ for any natural number $n$. Set $D=A \bowtie_{\tau} H$ and $\left[P_{n}\right]=1_{A} \otimes P_{n} \otimes 1_{H}$. Then $\left(D,\left\{\left[P_{n}\right]\right\}\right)$ is a almost cocommutative on $D_{(n)}$ iff

(ACO1): $\sum P^{\prime} y_{1} \otimes P^{\prime \prime} \otimes y_{2}=\sum y_{4} P^{\prime} \otimes P_{2}^{\prime \prime} \otimes y_{2} \tau\left(y_{1}, P_{1}^{\prime \prime}\right) \tau^{-1}\left(y_{3}, P_{3}^{\prime \prime}\right)$ for any $y \in H_{(n)}$; (ACO2): $\sum x_{2} \otimes P^{\prime} \otimes x_{1} P^{\prime \prime}=\sum x_{2} \otimes P_{2}^{\prime} \otimes P^{\prime \prime} x_{4} \tau\left(P_{1}^{\prime}, x_{1}\right) \tau^{-1}\left(P_{3}^{\prime}, x_{3}\right)$ for any $x \in A_{(n)}$. 
Proof. It is clear that $\left(D,\left\{\left[P_{n}\right]\right\}\right)$ is a almost cocommutative on $D_{(n)}$ iff the following holds:

$$
\begin{aligned}
& \sum x_{2} \otimes y_{4} P^{\prime} \otimes x_{1} P_{2}^{\prime \prime} \otimes y_{2} \tau\left(y_{1}, P_{1}^{\prime \prime}\right) \tau^{-1}\left(y_{3}, P_{3}^{\prime \prime}\right) \\
= & \sum x_{2} \otimes P_{2}^{\prime} y_{1} \otimes P^{\prime \prime} x_{4} \otimes y_{2} \tau\left(P_{1}^{\prime}, x_{1}\right) \tau^{-1}\left(P_{3}^{\prime}, x_{3}\right)
\end{aligned}
$$

for any $x \in A_{(n)}, y \in H_{(n)}$.

Assume that both (ACO1) and (ACO2) hold. See that

the left hand of $(2)$

$$
\begin{aligned}
& =\sum x_{2} \otimes P^{\prime} y_{1} \otimes x_{1} P^{\prime \prime} \otimes y_{2} \quad(\text { by }(\mathrm{ACO} 1)) \\
& =\text { the right hand of }(2) \quad(\text { by }(\mathrm{ACO} 2))
\end{aligned}
$$

for any $x \in A_{(n)}, y \in H_{(n)}$. That is, ( 2) holds.

Conversely, assume that (2) holds. Thus we have that

$$
\begin{aligned}
& \sum x_{2} \otimes P^{\prime} \otimes x_{1} P_{2}^{\prime \prime} \otimes \epsilon_{H}\left(1_{H}\right) \tau\left(1_{H}, P_{1}^{\prime \prime}\right) \tau^{-1}\left(1_{H}, P_{3}^{\prime \prime}\right) \\
= & \sum x_{2} \otimes P_{2}^{\prime} \otimes P^{\prime \prime} x_{4} \otimes \epsilon_{H}\left(1_{H}\right) \tau\left(P_{1}^{\prime}, x_{1}\right) \tau^{-1}\left(P_{3}^{\prime}, x_{3}\right)
\end{aligned}
$$

and

$$
\begin{aligned}
& \sum \epsilon_{A}\left(1_{A}\right) \otimes y_{4} P^{\prime} \otimes P_{2}^{\prime \prime} \otimes y_{2} \tau\left(y_{1}, P_{1}^{\prime \prime}\right) \tau^{-1}\left(y_{3}, P_{3}^{\prime \prime}\right) \\
= & \sum \epsilon_{A}\left(1_{A}\right) \otimes P_{2}^{\prime} y_{1} \otimes P^{\prime \prime} \otimes y_{2} \tau\left(P_{1}^{\prime}, 1_{A}\right) \tau^{-1}\left(P_{3}^{\prime}, 1_{A}\right)
\end{aligned}
$$

for any $x \in A_{(n)}, y \in H_{(n)}$. Consequently, (ACO1) and (ACO2) hold.

Lemma 3.6 Let $A=\oplus_{n=0}^{\infty} A_{n}$ and $H=\oplus_{n=0}^{\infty} H_{n}$ be two graded Hopf algebras with invertible antipodes. Let $\tau$ be a skew pairing on $(H \otimes A)$ and $P_{n}$ be a copairing of $\left(H_{(n)} \otimes\right.$ $\left.A_{(n)}\right)$ with $P_{n+1}=P_{n}+W_{n}$ and $W_{n} \in H_{(n+1)} \otimes A_{n+1}+H_{n+1} \otimes A_{(n+1)}$ for any natural number $n$. Set $D=A \bowtie_{\tau} H$. If $\tau\left(P_{n}^{\prime}, x\right) P_{n}^{\prime \prime}=x$ and $\tau\left(y, P_{n}^{\prime \prime}\right) P_{n}^{\prime}=y$ for any $x \in A_{(n)}, y \in H_{(n)}$, then $\left(D,\left\{\left[P_{n}\right]\right\}\right)$ is a local quasitriangular Hopf algebra.

Proof. It follows from Lemma 3.2 that $D=\oplus_{n=0}^{\infty} D_{n}$ is a Hopf algebra. Let $D_{n}=$ $\sum_{i+j=n} A_{i} \otimes H_{j}$. It is clear that $D=\oplus_{n=0}^{\infty} D_{n}$ is a graded coalgebra. We only need show that $\left(D,\left\{\left[P_{n}\right]\right\}\right)$ is almost cocommutative on $D_{(n)}$. Now fix $n$. For convenience, we denote $P_{n}$ by $P$ and $Q$ in following formulae. For any $x \in A_{i}, y \in H_{j}$ with $i+j \leq n$,

the right hand of $(\mathrm{ACO} 1)$

$$
\stackrel{\operatorname{by}(C P 1)}{=} \quad \sum y_{4} Q^{\prime} P^{\prime} \otimes Q_{1}^{\prime \prime} \otimes y_{2} \tau\left(y_{1}, P^{\prime \prime}\right) \tau^{-1}\left(y_{3}, Q_{2}^{\prime \prime}\right)
$$

by assumption $\sum y_{4} Q^{\prime} y_{1} \otimes Q_{1}^{\prime \prime} \otimes y_{2} \tau^{-1}\left(y_{3}, Q_{2}^{\prime \prime}\right)$ 


$$
\begin{aligned}
& \stackrel{\operatorname{by}(C P 1)}{=} \quad \sum y_{4} Q^{\prime} P^{\prime} y_{1} \otimes P^{\prime \prime} \otimes y_{2} \tau^{-1}\left(y_{3}, Q^{\prime \prime}\right) \\
& =\quad \sum y_{4} Q^{\prime} P^{\prime} y_{1} \otimes P^{\prime \prime} \otimes y_{2} \tau\left(S^{-1}\left(y_{3}\right), Q^{\prime \prime}\right) \\
& \text { by assumption } \sum y_{4} S^{-1}\left(y_{3}\right) P^{\prime} y_{1} \otimes P^{\prime \prime} \otimes y_{2} \\
& =\quad \sum P^{\prime} y_{1} \otimes P^{\prime \prime} \otimes y_{2} \\
& =\quad \text { the left hand of (ACO1) . }
\end{aligned}
$$

Similarly, we can show that $(\mathrm{ACO} 2)$ holds on $A_{(n)}$.

Theorem 6 Assume that $B$ is a finite dimensional Hopf algebra and $M$ is a finite dimenaional B-Hopf bimodule. Let $A:=T_{B}(M)^{\text {cop }}, H:=T_{B^{*}}^{c}\left(M^{*}\right)$ and $D=A \bowtie_{\tau} H$ with $\tau:=d_{H}(\phi \otimes i d) C_{H, A}$. Then $\left(\left(T_{B}(M)\right)^{\text {cop }} \bowtie_{\tau} T_{B^{*}}^{c}\left(M^{*}\right),\left\{R_{n}\right\}\right)$ is a local quasitriangular Hopf algebra. Here $P_{n}=\left(i d \otimes \psi_{n}\right) b_{H_{(n)}}, R_{n}=\left[P_{n}\right]=1_{B} \otimes\left(i d \otimes \psi_{n}\right) b_{H_{(n)}} \otimes 1_{B^{*}}$, $\phi$ and $\psi_{n}$ are defined in Lemma 3.1.

Proof. By Lemma 3.4 (ii), $A$ and $H$ have invertible antipodes. Assume that $e_{1}^{(i)}, e_{2}^{(i)}, \cdots, e_{n_{i}}^{(i)}$ is a basis of $H_{i}$ and $e_{1}^{(i) *}, e_{2}^{(i) *}, \cdots, e_{n_{i}}^{(i) *}$ is a its dual basis in $\left(H_{i}\right)^{*}$. Then $\left\{e_{j}^{(i)} \mid i=\right.$ $\left.0,1,2, \cdots, n ; j=1,2, \cdots, n_{i}\right\}$ is a basis of $H_{(n)}$ and $\left\{e_{j}^{(i) *} \mid i=0,1,2, \cdots, n ; j=1,2, \cdots, n_{i}\right\}$ is its dual basis in $\left(H_{(n)}\right)^{*}$. Thus $b_{H_{(n)}}=\sum_{i=0}^{n} \sum_{j=1}^{n_{i}} e_{j}^{(i)} \otimes e_{j}^{(i) *}$. See that

$$
\begin{aligned}
P_{n+1} & =\sum_{i=0}^{n} \sum_{j=1}^{n_{i}} e_{j}^{(i)} \otimes \psi_{n+1}\left(e_{j}^{(i) *}\right)+\sum_{j=1}^{n_{n+1}} e_{j}^{(n+1)} \otimes \psi_{n+1}\left(e_{j}^{(n+1) *}\right) \\
& =P_{n}+\sum_{j=1}^{n_{n+1}} e_{j}^{(n+1)} \otimes \psi_{n+1}\left(e_{j}^{(n+1) *}\right)
\end{aligned}
$$

Obviously, $\sum_{j=1}^{n_{n+1}} e_{j}^{(n+1)} \otimes \psi_{n+1}\left(e_{j}^{(n+1) *}\right) \in H_{n+1} \otimes A_{n+1}$. It is clear that $P_{n}$ is a copairing on $H_{(n)} \otimes A_{(n)}$ and $\tau$ is a skew pairing on $H \otimes A$ with $\sum \tau\left(P_{n}^{\prime}, x\right) P_{n}^{\prime \prime}=x$ and $\sum \tau\left(y, P_{n}^{\prime \prime}\right) P_{n}^{\prime}=y$ for any $x \in A_{(n)}, y \in H_{(n)}$. We complete the proof by Lemma 3.6.

Note that $R_{n+1}=R_{n}+W_{n}$ with $W_{n} \in D_{n+1} \otimes D_{n+1}$ in the above theorem.

\section{Quiver Hopf Algebras}

A quiver $Q=\left(Q_{0}, Q_{1}, s, t\right)$ is an oriented graph, where $Q_{0}$ and $Q_{1}$ are the sets of vertices and arrows, respectively; $s$ and $t$ are two maps from $Q_{1}$ to $Q_{0}$. For any arrow $a \in Q_{1}$, $s(a)$ and $t(a)$ are called its start vertex and end vertex, respectively, and $a$ is called an arrow from $s(a)$ to $t(a)$. For any $n \geq 0$, an $n$-path or a path of length $n$ in the quiver $Q$ is an ordered sequence of arrows $p=a_{n} a_{n-1} \cdots a_{1}$ with $t\left(a_{i}\right)=s\left(a_{i+1}\right)$ for all $1 \leq i \leq n-1$. Note that a 0 -path is exactly a vertex and a 1-path is exactly an arrow. In this case, we define $s(p)=s\left(a_{1}\right)$, the start vertex of $p$, and $t(p)=t\left(a_{n}\right)$, the end vertex of $p$. For 
a 0 -path $x$, we have $s(x)=t(x)=x$. Let $Q_{n}$ be the set of $n$-paths, $Q_{(n)}$ be the set of $i$-paths with $i \leq n$ and $Q_{\infty}$ be the set of all paths in $Q$. Let ${ }^{y} Q_{n}^{x}$ denote the set of all $n$-paths from $x$ to $y, x, y \in Q_{0}$. That is, ${ }^{y} Q_{n}^{x}=\left\{p \in Q_{n} \mid s(p)=x, t(p)=y\right\}$. A quiver $Q$ is finite if $Q_{0}$ and $Q_{1}$ are finite sets.

Let $G$ be a group. Let $\mathcal{K}(G)$ denote the set of conjugate classes in $G . r=\sum_{C \in \mathcal{K}(G)} r_{C} C$ is called a ramification (or ramification data) of $G$, if $r_{C}$ is the cardinal number of a set for any $C \in \mathcal{K}(G)$. We always assume that the cardinal number of the set $I_{C}(r)$ is $r_{C}$. Let $\mathcal{K}_{r}(G):=\left\{C \in \mathcal{K}(G) \mid r_{C} \neq 0\right\}=\left\{C \in \mathcal{K}(G) \mid I_{C}(r) \neq \emptyset\right\}$.

Let $G$ be a group. A quiver $Q$ is called a quiver of $G$ if $Q_{0}=G$ (i.e., $Q=\left(G, Q_{1}, s, t\right)$ ). If, in addition, there exists a ramification $r$ of $G$ such that the cardinal number of ${ }^{y} Q_{1}^{x}$ is equal to $r_{C}$ for any $x, y \in G$ with $x^{-1} y \in C \in \mathcal{K}(G)$, then $Q$ is called a Hopf quiver with respect to the ramification data $r$. In this case, there is a bijection from $I_{C}(r)$ to ${ }^{y} Q_{1}^{x}$. Denote by $(Q, G, r)$ the Hopf quiver of $G$ with respect to $r$.

$e$ denotes the unit element of $G .\left\{p_{g} \mid g \in G\right\}$ denotes the dual basis of $\{g \mid g \in G\}$ of finite group algebra $k G$.

Let $Q=\left(G, Q_{1}, s, t\right)$ be a quiver of a group $G$. Then $k Q_{1}$ becomes a $k G$-bicomodule under the natural comodule structures:

$$
\delta^{-}(a)=t(a) \otimes a, \quad \delta^{+}(a)=a \otimes s(a), \quad a \in Q_{1},
$$

called an arrow comodule, written as $k Q_{1}^{c}$. In this case, the path coalgebra $k Q^{c}$ is exactly isomorphic to the cotensor coalgebra $T_{k G}^{c}\left(k Q_{1}^{c}\right)$ over $k G$ in a natural way (see [3] and [5]). We will regard $k Q^{c}=T_{k G}^{c}\left(k Q_{1}^{c}\right)$ in the following. Moreover, $k Q_{1}$ becomes a $(k G)^{*}$ bimodule with the module structures defined by

$$
p \cdot a:=\langle p, t(a)\rangle a, \quad a \cdot p:=\langle p, s(a)\rangle a, \quad p \in(k G)^{*}, a \in Q_{1},
$$

written as $k Q_{1}^{a}$, called an arrow module. Therefore, we have a tensor algebra $T_{(k G)^{*}}\left(k Q_{1}^{a}\right)$. Note that the tensor algebra $T_{(k G)^{*}}\left(k Q_{1}^{a}\right)$ of $k Q_{1}^{a}$ over $(k G)^{*}$ is exactly isomorphic to the path algebra $k Q^{a}$. We will regard $k Q^{a}=T_{(k G)^{*}}\left(k Q_{1}^{a}\right)$ in the following.

Lemma 4.1 (see [5, Theorem 3.3], and [6, Theorem 3.1]) Let $Q$ be a quiver over group $G$. Then the following statements are equivalent:

(i) $Q$ is a Hopf quiver.

(ii) Arrow comodule $k Q_{1}^{c}$ admits a $k G$-Hopf bimodule structure.

If $Q$ is finite, then the above statements are also equivalent to the following:

(iii) Arrow module $k Q_{1}^{a}$ admits a $(k G)^{*}$-Hopf bimodule structure.

Assume that $Q$ is a Hopf quiver. It follows from Lemma 4.1 that there exist a left $k G$ - module structure $\alpha^{-}$and a right $k G$ - module structure $\alpha^{+}$on arrow comodule $\left(k Q_{1}^{c}, \delta^{-}, \delta^{+}\right)$such that $\left(k Q_{1}^{c}, \alpha^{-}, \alpha^{+}, \delta^{-}, \delta^{+}\right)$becomes a $k G$-Hopf bimodule, called a 
co-arrow Hopf bimodule. We obtain two graded Hopf algebras $T_{k G}\left(k Q_{1}^{c}\right)$ and $T_{k G}^{c}\left(k Q_{1}^{c}\right)$, called semi-path Hopf algebra and co-path Hopf algebra, written $k Q^{s}$ and $k Q^{c}$, respectively.

Assume that $Q$ is a finite Hopf quiver. Dually, it follows from Lemma 4.1 that there exist a left $(k G)^{*}$-comodule structure $\delta^{-}$and a right $(k G)^{*}$-comodule structure $\delta^{+}$on arrow module $\left(k Q_{1}^{a}, \alpha^{-}, \alpha^{+}\right)$such that $\left(k Q_{1}^{a}, \alpha^{-}, \alpha^{+}, \delta^{-}, \delta^{+}\right)$becomes a $(k G)^{*}$-Hopf bimodule, called a arrow Hopf bimodule. We obtain two graded Hopf algebras $T_{(k G)^{*}}\left(k Q_{1}^{a}\right)$ and $T_{(k G)^{*}}^{c}\left(k Q_{1}^{a}\right)$, called path Hopf algebra and semi-co-path Hopf algebra, written $k Q^{a}$ and $k Q^{s c}$, respectively.

From now on, we assume that $Q$ is a finite Hopf quiver on finite group $G$. Let $\xi_{k Q_{1}^{a}}$ denote the linear map from $k Q_{1}^{a}$ to $\left(k Q_{1}^{c}\right)^{*}$ by sending $a$ to $a^{*}$ for any $a \in Q_{1}$ and $\xi_{k Q_{1}^{c}}$ denote the linear map from $k Q_{1}^{c}$ to $\left(k Q_{1}^{a}\right)^{*}$ by sending $a$ to $a^{*}$ for any $a \in Q_{1}$. It is easy to check the following.

Lemma 4.2 (i) If $\left(M, \alpha^{-}, \alpha^{+}, \delta^{-}, \delta^{+}\right)$is a finite dimensional B-Hopf bimodule and $B$ is a finite dimensional Hopf algebra, then $\left(M^{*}, \delta^{-*}, \delta^{+*}, \alpha^{-*}, \alpha^{+*}\right)$ is a $B^{*}$-Hopf bimodule.

(ii) If $\left(k Q_{1}^{c}, \alpha^{-}, \alpha^{+}, \delta^{-}, \delta^{+}\right)$is a co-arrow Hopf bimodule, then there exist unique left $(k G)^{*}$-comodule operation $\delta_{k Q_{1}^{a}}^{-}$and right $(k G)^{*}$-comodule $\delta_{k Q_{1}^{a}}^{+}$such that $\left(k Q_{1}^{a}, \alpha_{k Q_{1}^{a}}^{-}, \alpha_{k Q_{1}^{a}}^{+}\right.$, $\left.\delta_{k Q_{1}^{a}}^{-}, \delta_{k Q_{1}^{a}}^{+}\right)$becomes a $(k G)^{*}$-Hopf bimodule and $\xi_{k Q_{1}^{a}}$ becomes a $(k G)^{*}$-Hopf bimodule isomorphism from $\left(k Q_{1}^{a}, \alpha_{k Q_{1}^{a}}^{-}, \alpha_{k Q_{1}^{+}}^{+}, \delta_{k Q_{1}^{a}}^{-}, \delta_{k Q_{1}^{a}}^{+}\right)$to $\left(\left(k Q_{1}^{c}\right)^{*}, \delta^{-*}, \delta^{+*}, \alpha^{-*}, \alpha^{+*}\right)$.

(iii) If $\left(k Q_{1}^{a}, \alpha^{-}, \alpha^{+}, \delta^{-}, \delta^{+}\right)$is a arrow Hopf bimodule, then there exist unique left $k G$ module operation $\alpha_{k Q_{1}^{c}}^{-}$and right $k G$-module $\alpha_{k Q_{1}^{c}}^{+}$such that $\left(k Q_{1}^{c}, \alpha_{k Q_{1}^{c}}^{-}, \alpha_{k Q_{1}^{c}}^{+}, \delta_{k Q_{1}^{c}}^{-}, \delta_{k Q_{1}^{c}}^{+}\right)$ become a $k G$-Hopf bimodule and $\xi_{k Q_{1}^{c}}$ becomes a $k G$-Hopf bimodule isomorphism from $\left(k Q_{1}^{c}, \alpha_{k Q_{1}^{c}}^{-}, \alpha_{k Q_{1}^{c}}^{+}, \delta_{k Q_{1}^{c}}^{-}, \delta_{k Q_{1}^{c}}^{+}\right)$to $\left(\left(k Q_{1}^{a}\right)^{*}, \delta^{-*}, \delta^{+*}, \alpha^{-*}, \alpha^{+*}\right)$.

(iv) $\xi_{k Q_{1}^{a}}$ is a $(k G)^{*}$-Hopf bimodule isomorphism from $\left(k Q_{1}^{a}, \alpha_{k Q_{1}^{a}}^{-}, \alpha_{k Q_{1}^{a}}^{+}, \delta_{k Q_{1}^{a}}^{-}, \delta_{k Q_{1}^{a}}^{+}\right)$to $\left(\left(k Q_{1}^{c}\right)^{*}, \delta_{k Q_{1}^{c}}^{-}, \delta_{k Q_{1}^{c}}^{+}, \alpha_{k Q_{1}^{c}}^{-}, \alpha_{k Q_{1}^{+}}^{+}\right)$iff $\xi_{k Q_{1}^{c}}$ becomes a $k G$-Hopf bimodule isomorphism from $\left(k Q_{1}^{c}, \alpha_{k Q_{1}^{c}}^{-}, \alpha_{k Q_{1}^{c}}^{+}, \delta_{k Q_{1}^{c}}^{-}, \delta_{k Q_{1}^{c}}^{+}\right)$to $\left(\left(k Q_{1}^{a}\right)^{*}, \delta_{k Q_{1}^{a}}^{-}, \delta_{k Q_{1}^{a}}^{+}, \alpha_{k Q_{1}^{a}}^{-}, \alpha_{k Q_{1}^{+}}^{+}\right)$.

Let $B$ be a Hopf algebra and ${ }_{B}^{B} \mathcal{M}_{B}^{B}$ denote the category of $B$-Hopf bimodules. Let $G \mathcal{H}$ opf denote the category of graded Hopf algebras. Define $T_{B}(\psi)=: T_{B}\left(\iota_{0}, \iota_{1} \psi\right)$ and $T_{B}^{c}(\psi):=T_{B}^{c}\left(\pi_{0}, \psi \pi_{1}\right)$ for any $B$-Hopf bimodule homomorphism $\psi$.

Lemma 4.3 Let $B$ be a Hopf algebras. Then $T_{B}$ and $T_{B}^{c}$ are two functors from ${ }_{B}^{B} \mathcal{M}_{B}^{B}$ to $G \mathcal{H}$ opf.

Proof. (i) If $\psi$ is a $B$-Hopf bimodule homomorphism from $M$ to $M^{\prime}$, then $T_{B}\left(\iota_{0}, \iota_{1} \psi\right)$ is a graded Hopf algebra homomorphism from $T_{B}(M)$ to $T_{B}\left(M^{\prime}\right)$. Indeed, let $\Phi:=$ $T_{B}\left(\iota_{0}, \iota_{1} \psi\right)$. Then both $\Delta_{T_{B^{\prime}}\left(M^{\prime}\right)} \Phi$ and $(\Phi \otimes \Phi) \Delta_{T_{B}(M)}$ are graded algebra maps from $T_{B}(M)$ to $T_{B}\left(M^{\prime}\right) \otimes T_{B}\left(M^{\prime}\right)$. Now we show that $\Delta_{T_{B}\left(M^{\prime}\right)} \Phi=(\Phi \otimes \Phi) \Delta_{T_{B}(M)}$. Considering 
that $\Phi$ is an algebra homomorphism, we only have to show that $\Delta_{T_{B}\left(M^{\prime}\right)} \Phi \iota_{0}=(\Phi \otimes$ $\Phi) \Delta_{T_{B}(M)} \iota_{0}$ and $\Delta_{T_{B}\left(M^{\prime}\right)} \Phi \iota_{1}=(\Phi \otimes \Phi) \Delta_{T_{B}(M)} \iota_{1}$. Obviously, the first equation holds. For the second equation, see

$$
\begin{aligned}
\Delta_{T_{B^{\prime}}\left(M^{\prime}\right)} \Phi \iota_{1} & =\Delta_{T_{B^{\prime}}\left(M^{\prime}\right)} \iota_{1} \psi \\
& =\left(\iota_{0} \otimes \iota_{1}\right) \delta_{M^{\prime}}^{-} \psi+\left(\iota_{1} \otimes \iota_{0}\right) \delta_{M^{\prime}}^{+} \psi \\
& =\left(\iota_{0} \otimes \iota_{1}\right)(i d \otimes \psi) \delta_{M}^{-}+\left(\iota_{1} \otimes \iota_{0}\right)(\psi \otimes i d) \delta_{M}^{+} \\
& =\left(\iota_{0} \otimes \iota_{1} \psi\right) \delta_{M}^{-}+\left(\iota_{1} \psi \otimes \iota_{0}\right) \delta_{M}^{+} \\
& =(\Phi \otimes \Phi)\left(\iota_{0} \otimes \iota_{1}\right) \delta_{M}^{-}+(\Phi \otimes \Phi)\left(\iota_{1} \otimes \iota_{0}\right) \delta_{M}^{+} \\
& =(\Phi \otimes \Phi)\left[\left(\iota_{0} \otimes \iota_{1}\right) \delta_{M}^{-}+\left(\iota_{1} \otimes \iota_{0}\right) \delta_{M}^{+}\right] \\
& =(\Phi \otimes \Phi) \Delta_{T_{B}(M)} \iota_{1} .
\end{aligned}
$$

Consequently, $T_{B}\left(\iota_{0}, \iota_{1} \psi\right)$ is a graded Hopf algebra homomorphisms.

(ii) If $\psi$ is a $B$-Hopf bimodule homomorphism from $M$ to $M^{\prime}$, then $T_{B}^{c}\left(\pi_{0}, \psi \pi_{1}\right)$ is graded Hopf algebra homomorphism from $T_{B}^{c}(M)$ to $T_{B}^{c}\left(M^{\prime}\right)$. Indeed, let $\Psi:=T_{B}^{c}\left(\pi_{0}, \psi \pi_{1}\right)$. Then both $\Psi \mu_{T_{B}^{c}(M)}$ and $\mu_{T_{B}^{c}\left(M^{\prime}\right)}(\Psi \otimes \Psi)$ are graded coalgebra maps from $T_{B}^{c}(M) \otimes T_{B}^{c}(M)$ to $T_{B}^{c}\left(M^{\prime}\right)$. Since $T_{B}^{c}(M) \otimes T_{B}^{c}(M)$ is a graded coalgebra, $\operatorname{corad}\left(T_{B}^{c}(M) \otimes T_{B}^{c}(M)\right) \subseteq$ $\left(T_{B}^{c}(M) \otimes T_{B}^{c}(M)\right)_{0}=\iota_{0}(B) \otimes \iota_{0}(B)$. It follows that $\left(\pi_{1} \Psi \mu_{T_{B}^{c}(M)}\right)\left(\operatorname{corad}\left(T_{B}^{c}(M) \otimes T_{B}^{c}(M)\right)\right)=$ 0 . Thus by the universal property of $T_{B}^{c}\left(M^{\prime}\right)$, in order to prove $\Psi \mu_{T_{B}^{c}(M)}=\mu_{T_{B}^{c}\left(M^{\prime}\right)}(\Psi \otimes \Psi)$, we only need to show $\pi_{n} \Psi \mu_{T_{B}^{c}(M)}=\pi_{n} \mu_{T_{B}^{c}\left(M^{\prime}\right)}(\Psi \otimes \Psi)$ for $n=0,1$. However, this follows from a straightforward computation dual to Part (i). Furthermore, one can see $\Psi(1)=1$. Hence $\Psi$ is an algebra map, and so a Hopf algebra map.

(iii) It is straightforward to check $T_{B}(\psi) T_{B}\left(\psi^{\prime}\right)=T_{B}\left(\psi \psi^{\prime}\right)$ and $T_{B}^{c}(\psi) T_{B}^{c}\left(\psi^{\prime}\right)=T_{B}^{c}\left(\psi \psi^{\prime}\right)$ for $B$-Hopf bimodule homomorphisms $\psi: M^{\prime} \rightarrow M^{\prime \prime}$ and $\psi^{\prime}: M \rightarrow M^{\prime}$.

(iv) $T_{B}\left(i d_{M}\right)=i d_{T_{B}(M)}$ and $T_{B}^{c}\left(i d_{M}\right)=i d_{T_{B}^{c}(M)}$.

Lemma 4.4 If $\psi$ is a Hopf algebra isomorphism from $B$ to $B^{\prime}$ and $\left(M, \alpha^{-}, \alpha^{+}, \delta^{-}, \delta^{+}\right)$ is a B-Hopf bimodule, then $\left(M, \alpha^{-}\left(\psi^{-1} \otimes i d_{M}\right), \alpha^{+}\left(i d_{M} \otimes \psi^{-1}\right),\left(\psi \otimes i d_{M}\right) \delta^{-},\left(i d_{M} \otimes \psi\right) \delta^{+}\right)$ is a $B^{\prime}$-Hopf bimodule. Furthermore $T_{B}\left(\iota_{0} \psi, \iota_{1}\right)$ and $T_{B}^{c}\left(\psi \pi_{0}, \pi_{1}\right)$ are graded Hopf algebra isomorphisms from $T_{B}(M)$ to $T_{B^{\prime}}(M)$ and from $T_{B}^{c}(M)$ to $T_{B^{\prime}}^{c}(M)$, respectively.

By Lemma 4.3 and Lemma 4.2 (ii) and (iii), $T_{(k G)^{*}}\left(\iota_{0}, \iota_{1} \xi_{k Q_{1}^{a}}\right)$ and $T_{k G}^{c}\left(\pi_{0}, \xi_{k Q_{1}^{c}} \pi_{1}\right)$ are graded Hopf algebra isomorphisms from $T_{(k G)^{*}}\left(k Q_{1}^{a}\right)$ to $T_{(k G)^{*}}\left(\left(k Q_{1}^{c}\right)^{*}\right)$ and from $T_{k G}^{c}\left(k Q_{1}^{c}\right)$ to $T_{k G}^{c}\left(\left(k Q_{1}^{a}\right)^{*}\right)$, respectively. $T_{(k G)^{*}}^{c}\left(\pi_{0}, \xi_{k Q_{1}^{a}} \pi_{1}\right)$ and $T_{k G}\left(\iota_{0}, \iota_{1} \xi_{k Q_{1}^{c}}\right)$ are graded Hopf algebra isomorphisms from $T_{(k G)^{*}}^{c}\left(k Q_{1}^{a}\right)$ to $T_{(k G)^{*}}^{c}\left(\left(k Q_{1}^{c}\right)^{*}\right)$ and from $T_{k G}\left(k Q_{1}^{c}\right)$ to $T_{k G}\left(\left(k Q_{1}^{a}\right)^{*}\right)$, respectively. Furthermore, $\left(k Q_{1}^{a}, k Q_{1}^{c}\right),\left(k Q^{a}, k Q^{c}\right)$ and $\left(k Q^{s}, k Q^{s c}\right)$ are said to be arrow dual pairings.

Theorem 7 Assume that $(Q, G, r)$ is a finite Hopf quiver on finite group $G$. If $\left(k Q^{a}, k Q^{c}\right)$ and $\left(k Q^{s}, k Q^{s c}\right)$ are said to be arrow dual pairings, then 
(i) $\left(\left(k Q^{a}\right)^{c o p} \bowtie_{\tau} k Q^{c},\left\{R_{n}\right\}\right)$ is a local quasitriangular Hopf algebra. Here

$$
R_{n}=\sum_{g \in G} p_{e} \otimes g \otimes p_{g} \otimes e+\sum_{q \in Q_{(n)}, q \notin G} p_{e} \otimes q \otimes q \otimes e
$$

and $\tau(a, b)=\delta_{a, b}$, for any two paths $a$ and $b$ in $Q$, where $\delta_{a, b}$ is the Kronecker symbol.

(ii) There exist $\tau$ and $\left\{R_{n}\right\}$ such that $\left(\left(k Q^{s}\right)^{c o p} \bowtie_{\tau} k Q^{s c},\left\{R_{n}\right\}\right)$ becomes a local quasitriangular Hopf algebra.

Proof. (i) Let $B:=(k G)^{*}$ and $M:=k Q_{1}^{a}$. Thus $T_{B}(M)=k Q^{a}$. Since $\left(k Q_{1}^{a}, k Q_{1}^{c}\right)$ is arrow dual pairing, $\xi_{k Q_{1}^{c}}$ is a $k G$-Hopf bimodule isomorphism from $k Q_{1}^{c}$ to $\left(k Q_{1}^{a}\right)^{*}$ by Lemma 4.2. See that

$$
\begin{aligned}
T_{B^{*}}^{c}\left(M^{*}\right) & =T_{(k G)^{* *}}^{c}\left(\left(k Q_{1}^{a}\right)^{*}\right) \\
& \stackrel{\nu_{1}}{\cong} T_{k G}^{c}\left(\left(k Q_{1}^{a}\right)^{*}\right) \quad(\text { by Lemma } 4.4) \\
& \stackrel{\nu_{2}}{\cong} T_{k G}^{c}\left(k Q_{1}^{c}\right) \quad(\text { by Lemma 4.2 and Lemma 4.3) } \\
& =k Q^{c},
\end{aligned}
$$

where $\nu_{1}=T_{k G}^{c}\left(\sigma_{k G}^{-1} \pi_{0}, \pi_{1}\right), \nu_{2}=T_{(k G)^{* *}}^{c}\left(\pi_{0},\left(\xi_{k Q_{1}^{c}}\right)^{-1} \pi_{1}\right)$.

Let $H=T_{B^{*}}^{c}\left(M^{*}\right)$ and $A=T_{B}(M)^{c o p}$. By Theorem 6, $\left(\left(k Q^{a}\right)^{c o p} \bowtie_{\tau} k Q^{c},\left\{R_{n}\right\}\right)$ is a local quasitriangular Hopf algebra. Here $\tau=d_{H}(\phi \otimes i d) C_{H, A}\left(\left(\nu_{2} \nu_{1}\right)^{-1} \otimes i d_{A}\right) ; R_{n}=$ $\left(i d_{A} \otimes \nu_{2} \nu_{1} \otimes i d_{A} \otimes \nu_{2} \nu_{1}\right)\left(1_{B} \otimes\left(i d \otimes \psi_{n}\right) b_{H_{(n)}} \otimes 1_{B^{*}}\right), \phi$ and $\psi_{n}$ are defined in Lemma 3.1. We have to show that they are the same as in this theorem. That is,

$$
\begin{gathered}
d_{H}(\phi \otimes i d) C_{H, A}\left(\left(\nu_{2} \nu_{1}\right)^{-1} \otimes i d_{A}\right)(a \otimes b)=\delta_{a, b} \\
\left(1_{B} \otimes b_{H_{(n)}} \otimes 1_{B^{*}}\right)=\left(i d_{A} \otimes\left(\nu_{2} \nu_{1}\right)^{-1} \otimes \phi_{n} \otimes\left(\nu_{2} \nu_{1}\right)^{-1}\right) \\
\left(\sum_{g \in G} p_{e} \otimes g \otimes p_{g} \otimes e+\sum_{q \in Q_{(n)}, q \notin G} \quad p_{e} \otimes q \otimes q \otimes e\right) .
\end{gathered}
$$

If $b=b_{n} b_{n-1} \cdots b_{1}$ is a $n$-path in $k Q^{c}$ with $b_{i} \in Q_{1}$ for $i=1,2, \cdots, n$, then

$$
\left(\nu_{2} \nu_{1}\right)^{-1}(b)=b_{n}^{*} \otimes b_{n-1}^{*} \otimes \cdots \otimes b_{1}^{*} .
$$

If $b \in G$, then $\left(\nu_{2} \nu_{1}\right)^{-1}(b)=\sigma_{k G}(b)$. Consequently, for any $a, b \in Q_{\infty}$ with $b \in k Q^{c}$ and $a \in k Q^{a}$, we have

$$
\begin{aligned}
& d_{H}(\phi \otimes i d) C_{H, A}\left(\left(\nu_{2} \nu_{1}\right)^{-1} \otimes i d_{A}\right)(a \otimes b) \\
= & d_{H}\left(\phi(a) \otimes\left(\nu_{2} \nu_{1}\right)^{-1}(b)\right) \\
= & \left\{\begin{array}{ll}
<\phi(a), \sigma_{k G}(b)>=<\sigma_{k G}(b), a>=\delta_{a, b}, & \text { when } b \in Q_{0} \\
<\left(\nu_{2} \nu_{1}\right)^{-1}, a>=\delta_{a, b} & (\text { by }(7)),
\end{array} .\right.
\end{aligned}
$$


Thus (5) holds. Note that $Q_{n}$ is a basis of not only $\left(k Q^{a}\right)_{n}$ but also $\left(k Q^{c}\right)_{n}$ for $n>0$. By (7), $\left\{\phi(q) \mid q \in Q_{n}\right\}$ is the dual basis of $\left\{\left(\nu_{2} \nu_{1}\right)^{-1}(q) \mid q \in Q_{n}\right\}$ for any $n>0$. Consequently, (6) holds.

(ii) Let $B:=k G$ and $M:=k Q_{1}^{c}$. Thus $T_{B}(M)=k Q^{s}$. Since $\left(k Q_{1}^{a}, k Q_{1}^{c}\right)$ is arrow dual pairing, $\xi_{k Q_{1}^{a}}$ is a $(k G)^{*}$-Hopf bimodule isomorphism from $k Q_{1}^{a}$ to $\left(k Q_{1}^{c}\right)^{*}$ by Lemma 4.2. Thus

$$
\begin{aligned}
T_{B^{*}}^{c}\left(M^{*}\right) & =T_{(k G)^{*}}^{c}\left(\left(k Q_{1}^{c}\right)^{*}\right) \\
& \stackrel{\nu_{3}}{\cong} T_{(k G)^{*}}^{c}\left(k Q_{1}^{a}\right) \quad(\text { by Lemma } 4.2 \text { and Lemma } 4.3) \\
& =k Q^{s c}
\end{aligned}
$$

where $\nu_{3}=T_{(k G)^{*}}^{c}\left(\pi_{0},\left(\xi_{k Q_{1}^{a}}\right)^{-1} \pi_{1}\right)$. By Theorem 6 , the double cross product of $T_{B}(M)^{\text {cop }}$ and $T_{B^{*}}\left(M^{*}\right)$ is a local quasitriangular Hopf algebra. Consequently, so is the double cross product of $\left(k Q^{s}\right)^{c o p}$ and $k Q^{s c}$.

Note (LQT4') holds in above theorem.

Example 4.5 Let $G=\mathbf{Z}_{2}=(g)=\{e, g\}$ be the group of order 2 with char $k \neq 2, X$ and $Y$ be respectively the set of arrows from $g^{0}$ to $g^{0}$ and the set of arrows from $g$ to $g$, and $|X|=|Y|=3$. The quiver $Q$ is a Hopf quiver with respect to ramification $r=r_{\{e\}}\{e\}$ with $r_{\{e\}}=3$. Let $\chi_{e}^{(i)} \in \mathbf{Z}_{2}$ and $a_{y, x}^{(i)}$ denote the arrow from $x$ to $y$ for $i=1,2,3$. Define $\delta^{-}\left(a_{x, x}^{(i)}\right)=x \otimes a_{x, x}^{(i)}, \delta^{+}\left(a_{x, x}^{(i)}\right)=a_{x, x}^{(i)} \otimes x, g \cdot a_{x, x}^{(i)}=a_{g x, g x}^{(i)}, a_{e, e}^{(i)} \cdot g=\chi_{e}^{(i)}(g) a_{x g, x g}^{(i)}$ for $x \in G, i=1,2,3$. By [5], $k Q_{1}$ is a $k G$-Hopf bialgebra. Therefore, it follows from Theorem 7 that $\left(\left(k Q^{a}\right)^{c o p} \bowtie_{\tau} k Q^{c},\left\{R_{n}\right\}\right)$ is a local quasitriangular Hopf algebra and for every finite dimensional $\left(k Q^{a}\right)^{c o p} \bowtie_{\tau} k Q^{c}$-module $M, C^{\left\{R_{n}\right\}}$ is a solution of Yang-Baxter equations on $M$.

By the way, we obtain the relation between path algebras and path coalgebras by Theorem 5 .

Corollary 4.6 Let $Q$ be a finite quiver over finite group $G$. Then Path algebra $k Q^{a}$ is algebra isomorphic to subalgebra $\sum_{n=0}^{\infty}\left(\square_{k G}^{n} k Q_{1}^{c}\right)^{*}$ of $\left(k Q^{c}\right)^{*}$.

Proof. Let $A=(k G)^{*}$ and $M=k Q_{1}^{c}$. It is clear that $\xi_{k Q_{1}^{c}}$ is a $k G$ - bicomodule isomorphism from $k Q_{1}^{c}$ to $\left(k Q_{1}^{a}\right)^{*}$. See that

$$
\begin{aligned}
T_{B^{*}}^{c}\left(M^{*}\right) & =T_{(k G)^{* *}}^{c}\left(\left(k Q_{1}^{a}\right)^{*}\right) \\
& \stackrel{\nu_{1}}{\cong} T_{k G}^{c}\left(\left(k Q_{1}^{a}\right)^{*}\right) \\
& \stackrel{\nu_{2}}{\cong} T_{k G}^{c}\left(k Q_{1}^{c}\right) \\
& =k Q^{c}
\end{aligned}
$$


where $\nu_{1}=T_{k G}^{c}\left(\sigma_{k G}^{-1} \pi_{0}, \pi_{1}\right), \nu_{2}=T_{k G}^{c}\left(\pi_{0},\left(\xi_{k Q_{1}^{c}}\right)^{-1} \pi_{1}\right)$. Obviously, $\nu_{1}$ and $\nu_{2}$ are coalgebra isomorphism. Consequently, it follows from Theorem 5 that $k Q^{a}$ is algebra isomorphism to subalgebra $\sum_{n=0}^{\infty}\left(\square_{k G}^{n} k Q_{1}^{c}\right)^{*}$ of $\left(k Q^{c}\right)^{*}$.

Acknowledgement: The authors were financially supported by the Australian Research Council. S.Z thanks Department of Mathematics, University of Queensland for hospitality.

\section{References}

[1] M. Auslander, I. Reiten and S. O. Smal $\phi$, Representation theory of Artin algebras, Cambridge University Press, 1995.

[2] A.A. Belavin and V.G.Drinfel'd, solutions of the classical Yang-Baxter equations for simple Lie algebras, Functional anal. appl, 16(1982), No. 3, 159-180.

[3] W. Chin and S. Montgomery, Basic coalgebras, modular interfaces, AMS/IP Stud. Adv. Math. 4, Amer. Math. Soc., Providence, RI, 1997, pp.41-47.

[4] C. Cibils, A quiver quantum group, Commun. Math. Phys. 157(1993), 459-477.

[5] C. Cibils and M. Rosso, Hopf quivers, J. Alg. 254 (2002), 241-251.

[6] C. Cibils and M. Rosso, Algebres des chemins quantiques, Adv. Math. 125 (1997), 171-199.

[7] V. G. Drinfel'd. Quantum groups. In "Proceedings International Congress of Mathematicians, August 3-11, 1986, Berkeley, CA" pp. 798-820, Amer. Math. Soc., Providence, RI, 1987.

[8] S. Dascalescu, C. Nastasecu and S. Raianu, Hopf algebras: an introduction, Marcel Dekker Inc., 2001.

[9] Y. Doi and M. Takeuchi. Multiplication algebra by two-cocycles . Commun. Alg. 14 (1994), 5715-5731.

[10] S. Majid, Algebras and Hopf algebras in braided categories, Lecture Notes in Pure and Appl. Math. 158, Marcel Dekker, New York, 1994, pp. 55-105.

[11] S. Montgomery, Hopf algebras and their actions on rings, CBMS no.82, AMS, Providence, RI, 1993. 
[12] W. Nichols, Bialgebras of type one, Commun. Alg. 6 (1978), 1521-1552.

[13] R. S. Pierce, Associative algebras, GTM 88, Springer, 1982.

[14] G.Lusztig, Introduction to quantum groups, Progress in Math., 110, Birkhauser, Boston, MA, 1993.

[15] C.Kassel, Quantum Groups, GTM 155, Springer-Verlag, 19995.

[16] M. E. Sweedler, Hopf algebras, Benjamin, New York, 1969.

[17] C.N.Yang and M.L.Ge, eds., Braid group, knot theory and statistical mechanics, World scientific, Singapore, 1989.

[18] S. Zhang, H.-X. Chen, The double bicrossproducts in braided tensor categories, Commun. Alg. 29 (2001), 31-66. 